\title{
A REGRESSION CHARACTERIZATION OF INVERSE GAUSSIAN DISTRIBUTIONS AND APPLICATION TO EDF GOODNESS-OF-FIT TESTS
}

\author{
KHOAN T. DINH, NHU T. NGUYEN, and TRUC T. NGUYEN
}

Received 15 July 2002

\begin{abstract}
We give a new characterization of inverse Gaussian distributions using the regression of a suitable statistic based on a given random sample. A corollary of this result is a characterization of inverse Gaussian distribution based on a conditional joint density function of the sample. Application of this corollary as a transformation in the procedure to construct EDF (empirical distribution function) goodness-of-fit tests for inverse Gaussian distributions is also studied.
\end{abstract}

2000 Mathematics Subject Classification: 62E10, 62F03.

1. Introduction. A distribution is an inverse Gaussian distribution with parameters $m>0$ and $\lambda>0$, denoted $\operatorname{IG}(m, \lambda)$, if it has a density function given by

$$
f(x ; m, \lambda)= \begin{cases}\left(\frac{\lambda}{2 \pi x^{3}}\right)^{1 / 2} \exp \left\{-\frac{\lambda(x-m)^{2}}{2 m^{2} x}\right\} & \text { for } x>0 \\ 0 & \text { otherwise }\end{cases}
$$

(See Tweedie [11].)

The characteristic function of an $\operatorname{IG}(m, \lambda)$ distribution is

$$
\varphi(t)=\exp \left\{\frac{\lambda\left[1-\left(1-2 i m^{2} t \lambda^{-1}\right)^{1 / 2}\right]}{m}\right\} .
$$

Let $X_{j}, j=1, \ldots, n, n \geq 2$, be a random sample from an $\mathrm{IG}(m, \lambda)$ distribution. Then, the statistics $Y=\sum_{j=1}^{n} X_{j}$ and $Z=\sum_{j=1}^{n} X_{j}^{-1}-n^{2} Y^{-1}$ are jointly complete sufficient for $m$ and $\lambda . Y$ and $Z$ are independently distributed, $Y$ has an IG $\left(n m, n^{2} \lambda\right)$ distribution, and $\lambda Z$ has a chi-square distribution with $(n-1)$ degrees of freedom. Khatri [4] gave a characterization of the inverse Gaussian distributions based on the independence between $Y$ and $Z$, then Seshadri [9] 
gave several characterizations of the inverse Gaussian distributions based on the constant regression of several different statistics given $Y$. In this note, we give a characterization of the inverse Gaussian distributions based on the regression of a statistic given $Y$ and $Z$. The corollary of this result is a characterization of the inverse Gaussian distributions based on the conditional joint density function of $X_{1}, \ldots, X_{n-2}$, given $Y$ and $Z$. The result of this corollary can be used as a transformation in the procedure to construct EDF (empirical distribution function) goodness-of-fit tests for inverse Gaussian distributions.

2. Characterization results. The conditional joint density function of $X_{1}, \ldots, X_{n-2}$, given $Y=y>0, Z=z>0$, is

$$
\begin{aligned}
& f_{X_{1}, \ldots, X_{n-2} \mid Y, Z\left(x_{1}, \ldots, x_{n-2} \mid y, z\right)} \\
& \qquad\left\{\begin{array}{c}
\frac{2 \Gamma((n-1) / 2) y^{3 / 2}}{n \pi^{(n-1) / 2} \prod_{j=1}^{n-2} x_{j}^{3 / 2}\left(y-\sum_{j=1}^{n-2} x_{j}\right)^{1 / 2} z^{(n-1) / 2}} \\
\times\left[\left(y-\sum_{j=1}^{n-2} x_{j}\right)^{2}\left(z+n^{2} y^{-1}-\sum_{j=1}^{n-2} x_{j}^{-1}\right)-4\left(y-\sum_{j=1}^{n-2} x_{j}\right)\right]^{-1 / 2} \\
\text { for } \sum_{j=1}^{n-2} x_{j}<y,\left(y-\sum_{j=1}^{n-2} x_{j}\right)\left(z+n^{2} y^{-1}-\sum_{j=1}^{n-2} x_{j}^{-1}\right)<4, \\
\text { otherwise. }
\end{array}\right.
\end{aligned}
$$

From (2.1), the UMVUE of the density function at a point $x_{1}>0$ is given by

$f_{X_{1} \mid Y, Z\left(x_{1} \mid y, z\right)}$

$$
=\left\{\begin{array}{l}
\frac{(n-1) \Gamma((n-1) / 2)}{n \sqrt{\pi} \Gamma((n-2) / 2)} \\
\times \frac{y^{3 / 2}\left[z+n^{2} y^{-1}-x_{1}^{-1}-(n-1)^{2}\left(y-x_{1}\right)^{-1}\right]^{(n-2) / 2-1}}{x_{1}^{3 / 2}\left(y-x_{1}\right)^{3 / 2} z^{(n-1) / 2}} \\
\text { for } x_{1}<y, z+n^{2} y^{-1}-x_{1}^{-1}-(n-1)^{2}\left(y-x_{1}\right)^{-1}>0 \\
\text { otherwise }
\end{array}\right.
$$

where $y, z>0$. (See Chhikara and Folks [1].) 
Let $T=X_{1}\left[Z+n^{2} Y^{-1}-X_{1}^{-1}-(n-1)^{2}\left(Y-X_{1}\right)^{-1}\right] . E[T \mid Y, Z]$ can be computed in two different ways. On the one hand,

$$
\begin{aligned}
E[T \mid Y, Z] & =E\left[X_{1}\left[Z+n^{2} Y^{-1}-X_{1}^{-1}-(n-1)^{2}\left(Y-X_{1}\right)^{-1} \mid Y, Z\right]\right] \\
& =\frac{Y Z}{n}+(n-1)-(n-1)^{2} E\left[X_{1}\left(Y-X_{1}\right)^{-1} \mid Y, Z\right] \\
& =\frac{Y Z}{n}+n(n-1)-(n-1)^{2} Y E\left[\left(Y-X_{1}\right)^{-1} \mid Y, Z\right] .
\end{aligned}
$$

On the other hand, this expectation can be computed using the conditional density function of $X_{1}$ given by (2.2), and the following integral is taken on the support of this conditional density function:

$$
E[T \mid Y, Z]=\int t(x) f_{X_{1} \mid Y, Z(x)} d x=\int u d v
$$

where

$$
\begin{aligned}
u(x) & =\frac{n-1}{n} \times \frac{\Gamma((n-1) / 2) y^{3 / 2}\left[z+n^{2} y^{-1}-x^{-1}-(n-1)^{2}(y-x)^{-1}\right]^{(n-2) / 2}}{\sqrt{\pi} \Gamma((n-2) / 2) z^{(n-1) / 2-1}}, \\
d v & =\frac{d x}{x^{1 / 2}(y-x)^{3 / 2}} .
\end{aligned}
$$

Hence,

$$
v=\frac{2 x^{1 / 2}}{y(y-x)^{1 / 2}} .
$$

Using integration by parts,

$$
\begin{aligned}
E[T \mid Y, Z] & =-\left[\frac{n-2}{Y}\right] E\left[\left(Y-X_{1}\right)-(n-1)^{2} X_{1}^{2}\left(Y-X_{1}\right)^{-1} \mid Y, Z\right] \\
& =-n(n-1)(n-2)+(n-1)^{2}(n-2) Y E\left[\left(Y-X_{1}\right)^{-1} \mid Y, Z\right] .
\end{aligned}
$$

Comparing (2.3) and (2.7),

$$
E\left[\left(Y-X_{1}\right)^{-1} \mid Y, Z\right]=\frac{n Y^{-1}}{n-1}+\frac{Z}{n(n-1)^{3}} .
$$

In the following part, we construct a characterization of inverse Gaussian distributions based on regression (2.8).

If $X$ has an inverse Gaussian distribution with the characteristic function $\varphi(t)$ given by (1.2), then take logarithm of this characteristic function following three successive differentiations and several simplifications, then $\varphi(t)$ satisfies the differential equation

$$
\varphi^{\prime 4}(t)-3 \varphi(t) \varphi^{\prime 2}(t) \varphi^{\prime \prime}(t)-\varphi^{2}(t) \varphi^{\prime}(t) \varphi^{\prime \prime \prime}(t)+3 \varphi^{2}(t) \varphi^{\prime \prime 2}(t)=0 .
$$


Conversely, if $\varphi(t)$ is the characteristic function of a random variable $X$ with finite $E\left[X^{-1}\right]$ and $E\left[X^{3}\right]$, that is, a solution of the differential equation (2.9), then, by the continuity of a characteristic function using the reverse procedure for getting (2.9), this characteristic function is (1.2). Hence, the following result is obtained.

LEMMA 2.1. Let $X$ be a nonnegative random variable with a nondegenerate distribution $F$ and with finite $E\left[X^{-1}\right]$ and $E\left[X^{3}\right]$. Assume that $E[X]=m$ and $\operatorname{Var}(X)=m^{3} / \lambda$ for some positive numbers $m$ and $\lambda$, then $F$ is an $\operatorname{IG}(m, \lambda)$ if and only if its characteristic function is a solution of the differential equation (2.9).

The following theorem is the main result of this note.

THEOREM 2.2. Let $X_{j}, j=1, \ldots, n, n \geq 2$, be a random sample of $n$ nonnegative random variables from a nondegenerate distribution $F$ with finite $E[X]$ and $\operatorname{Var}(X)$. Then, $F$ is an inverse Gaussian distribution if and only if regression (2.8) holds.

Proof. We only need to show that if (2.8) holds, then $F$ is an inverse Gaussian distribution.

From (2.8),

$$
E\left[e^{i t Y}\left\{n(n-1)^{3}\left(Y-X_{1}\right)^{-1}-n^{3}(n-2) Y^{-1}-\sum_{j=1}^{n} X_{j}^{-1}\right\}\right]=0 .
$$

From the fact that $X$ is a random variable with finite $E\left[X^{-1}\right]$,

$$
E\left[X^{-1} e^{i t X}\right]=i \int_{-T}^{t} \varphi(u) d u+\int_{R} x^{-1} e^{-i T x} d F(x)
$$

for any constant $T$ such that $-T<t$, where $\varphi$ is the characteristic function of $X$ (Khatri [4]), then

$$
\begin{aligned}
I_{1}(t) & =E\left[e^{i t\left(Y-X_{1}\right)}\left(Y-X_{1}\right)^{-1}\right] \\
& =i \int_{-T}^{t} \varphi^{n-1}(u) d u+\int_{R} x^{-1} e^{-i T x} d F^{*(n-1)}(x), \\
I_{2}(t) & =E\left[e^{i t Y} Y^{-1}\right]=i \int_{-T}^{t} \varphi^{n}(u) d u+\int_{R} x^{-1} e^{-i T x} d F^{*(n)}(x), \\
I_{3}(t) & =E\left[e^{i t X} j X_{j}^{-1}\right]=i \int_{-T}^{t} \varphi(u) d u+\int_{R} x^{-1} e^{-i T x} d F(x),
\end{aligned}
$$

where $F^{*(k)}$ denotes the $k$ times convolution of $F$. Substitute (2.12) into (2.10), simplify, and differentiate three times, the differential equation (2.9) is obtained. Then by Lemma 2.1, $F$ is an inverse Gaussian distribution. 
The following characterization of inverse Gaussian distributions based on (2.1) or (2.2) can be obtained directly from Theorem 2.2. This result will be used as a transformation in the procedure to construct EDF goodness-of-fit tests for inverse Gaussian distributions. The application of this result is discussed in Section 3.

COROLLARY 2.3. Let $X_{j}, j=1, \ldots, n, n \geq 2$, be a random sample of nonnegative random variables from a nondegenerate distribution $F$ with finite $E\left[X^{-1}\right]$ and $E\left[X^{3}\right] . F$ is an inverse Gaussian distribution if and only if the conditional joint density function of $X_{1}, \ldots, X_{n-2}$, given $Y=y>0$ and $Z=z>0$, is (2.1), or the conditional density function of $X_{1}$, given $Y=y>0$ and $Z=z>0$, is (2.2).

3. Application to goodness-of-fit test. Let $X_{j}, j=1, \ldots, n, n \geq 2$, be a sample of nonnegative random variables from a nondegenerate distribution $F$ with finite $E\left[X^{-1}\right]$ and $E\left[X^{3}\right]$. To test whether $F$ is an inverse Gaussian distribution, by Corollary 2.3, it is to test the equivalent simple hypothesis that whether the conditional joint density of $X_{1}, \ldots, X_{n-2}$, given $Y=y>0$ and $Z=z>0$, is (1.1). The results of Rosenblatt [8] and then of Chhikara and Folks [1] are used to change the $X$ 's sample to the $U$ 's random sample from a distribution over the interval $(0,1)$, and the equivalent hypothesis now is whether the $U$ 's sample is from the uniform distribution over the interval $(0,1)$. Then, any EDF test statistics can be used (D'Agostino and Stephens [2]). Nguyen and Dinh [5] used this transformation and studied the first exact EDF goodness-of-fit tests for inverse Gaussian distributions. In their study, at some alternative distributions, and with reasonable, not large, sample sizes, the exact EDF goodness-of-fit tests based on this transformation behave pretty well comparing with the other approximate EDF goodness-of-fit tests. The other goodness-of-fit tests for inverse Gaussian distributions using EDF statistics were given by Edgeman et al. [3], O’Reilly and Rueda [6], and Pavur et al. [7]. For detailed references, see Seshadri [10].

\section{REFERENCES}

[1] R. S. Chhikara and J. L. Folks, Estimation of the inverse Gaussian distribution function, J. Amer. Statist. Assoc. 69 (1974), 250-254.

[2] R. B. D'Agostino and M. A. Stephens (eds.), Goodness-of-Fit Techniques, Statistics: Textbooks and Monographs, vol. 68, Marcel Dekker, New York, 1986.

[3] R. L. Edgeman, R. C. Scott, and R. J. Pavur, A modified Kolmogorov-Smirnov test for the inverse Gaussian density with unknown parameters, Comm. Statist. Simulation Comput. 17 (1988), no. 4, 1203-1212.

[4] C. G. Khatri, A characterization of the inverse Gaussian distribution, Ann. Math. Statist. 33 (1962), 800-803.

[5] T. T. Nguyen and K. T. Dinh, Exact EDF goodness-of-fit tests for inverse Gaussian distributions, to appear in Comm. Statist.-Simulation Comput.

[6] F. J. O'Reilly and R. Rueda, Goodness of fit for the inverse Gaussian distribution, Canad. J. Statist. 20 (1992), no. 4, 387-397. 
[7] R. J. Pavur, R. L. Edgeman, and R. C. Scott, Quadratic statistics for the goodnessof-fit test of the inverse Gaussian distribution, IEEE Trans. Reliab. 41 (1992), no. $1,118-123$.

[8] M. Rosenblatt, Remarks on a multivariate transformation, Ann. Math. Statistics 23 (1952), 470-472.

[9] V. Seshadri, The inverse Gaussian distribution: some properties and characterizations, Canad. J. Statist. 11 (1983), no. 2, 131-136.

[10] _ The Inverse Gaussian Distribution. Statistical Theory and Applications, Lecture Notes in Statistics, vol. 137, Springer-Verlag, New York, 1999.

[11] M. C. K. Tweedie, Statistical properties of inverse Gaussian distributions. I, Ann. Math. Statist. 28 (1957), 362-377.

Khoan T. Dinh: US Environmental Protection Agency (EPA), Washington, DC 20460, USA

Nhu T. Nguyen: Department of Mathematical Sciences, New Mexico State University, Las Cruces, NM 88003-8001, USA

Truc T. Nguyen: Department of Mathematics and Statistics, Bowling Green State University, Bowling Green, OH 43403-0221, USA 


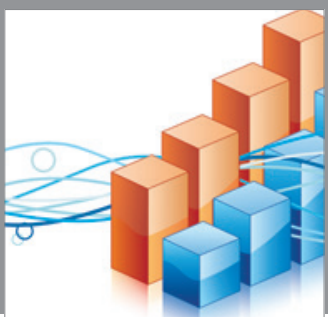

Advances in

Operations Research

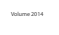

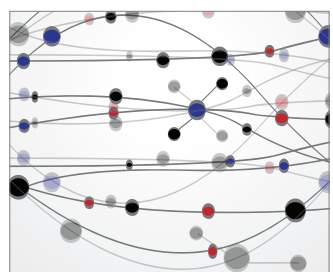

\section{The Scientific} World Journal
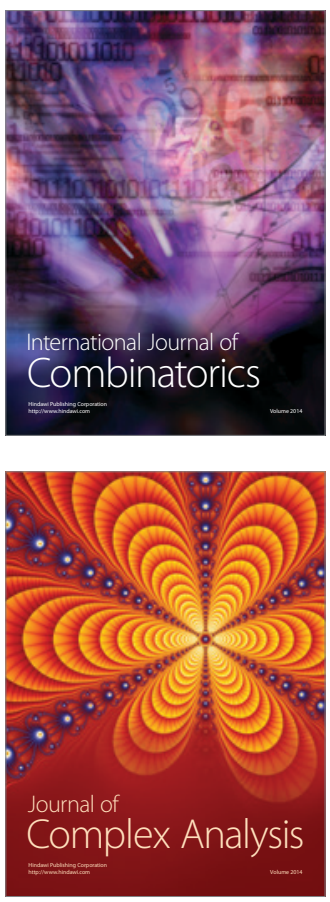

International Journal of

Mathematics and

Mathematical

Sciences
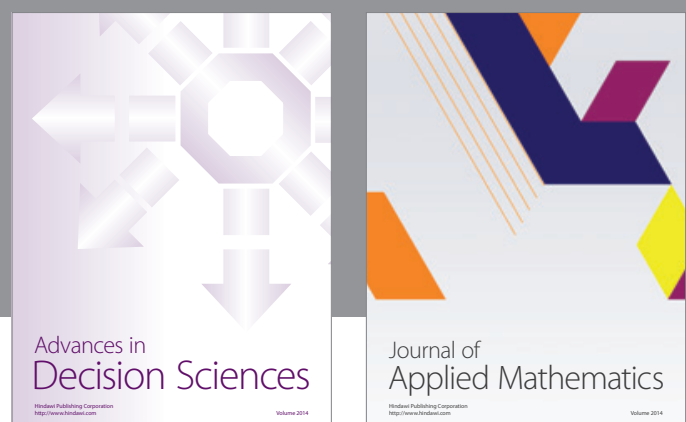

Journal of

Applied Mathematics
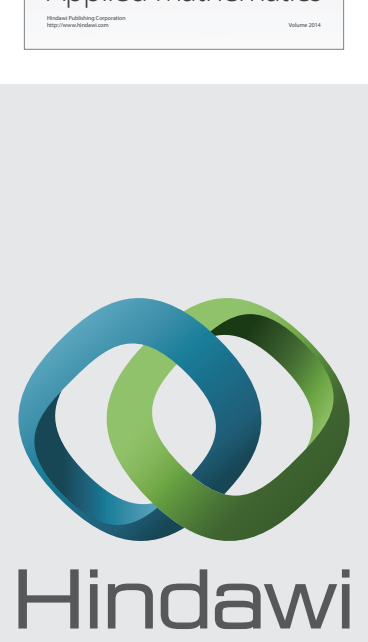

Submit your manuscripts at http://www.hindawi.com
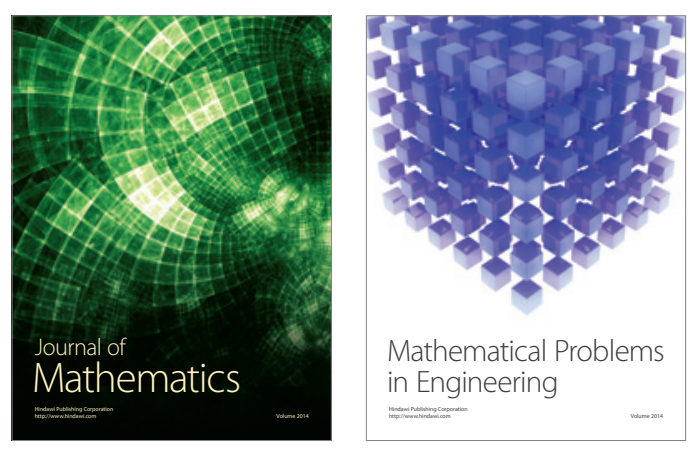

Mathematical Problems in Engineering
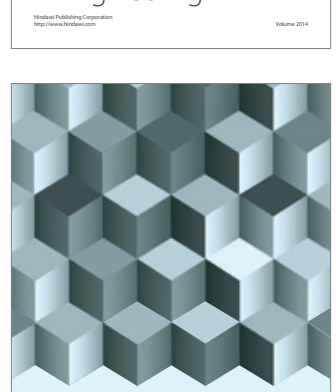

Journal of

Function Spaces
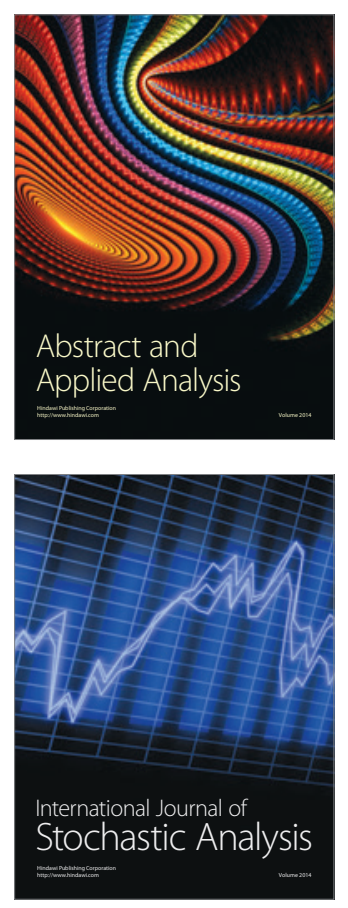

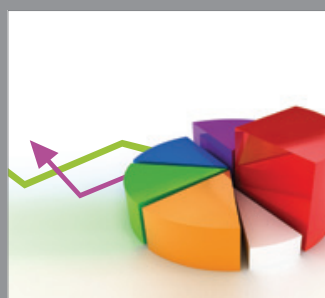

ournal of

Probability and Statistics

Promensencen
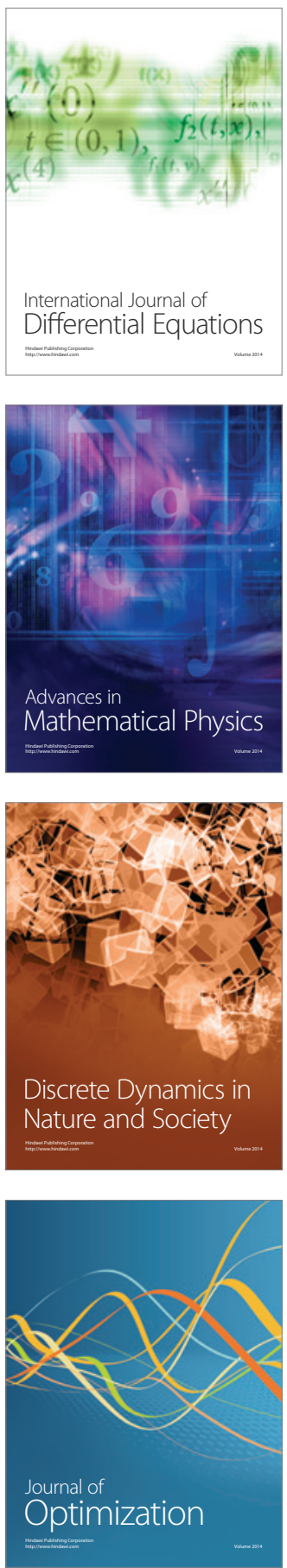